\title{
ON MONOTONE MULTIPLICATIVE FUNCTIONS
}

\section{MOSER AND J. LAMBEK}

A function $f(n)$, from positive integers to reals, which satisfies

$$
f(m n)=f(m) f(n), \quad(m, n)=1 ; \quad f(n) \not \equiv 0,
$$

is called weakly multiplicative or factorable. Although such functions are of central interest in number theory and have been studied in some detail in recent years, we have been unable to find the following result in the literature:

TheOREM. If $f(n)$ satisfies (1) and

$$
f(m) \geqq f(n) \quad \text { for } m \geqq n,
$$

then $f(n)=n^{k}$, where $k$ is a constant.

This theorem is similar to Ostrowski's lemma on Archimedean valuations. The main difficulty in proving it stems from the restriction $(m, n)=1$ in (1). It may be noted that, if (1) is relaxed to $f(m n)$ $\geqq f(m) f(n)$ or $f(m n) \leqq f(m) f(n)$, no such result holds. This may be seen from the counter examples $f(n)=n-1$ and $f(n)=n+1$. let

We proceed to the proof of the theorem. For a positive integer $a$

$$
\begin{aligned}
& R_{t}=a^{t}+a^{t-1}+\cdots+a+1, \\
& S_{t}=a^{t}-a^{t-1}-\cdots-a-1 \quad(a>1) .
\end{aligned}
$$

Clearly $\left(a, R_{t}\right)=\left(a, S_{t}\right)=1$, hence

$$
f\left(R_{t}\right) \geqq f\left(R_{t}-1\right)=f(a) f\left(R_{t-1}\right) \geqq \cdots \geqq(f(a))^{t} .
$$

Similarly

$$
f\left(S_{\imath}\right) \leqq(f(a))^{t}
$$

Given $n$, let $r$ be the integer determined by

$$
a^{r}<n \leqq a^{r+1},
$$

so that

$$
r<\log _{a} n \leqq r+1 .
$$

Now by (3), (4), (7), we have

$$
R_{r-1}<n
$$

Received by the editors July 16, 1952. 
and

$$
S_{r+2}>a^{r+2}-2 a^{r+1} \geqq a^{r+1} \geqq n \quad(a>2) .
$$

Now from (5) to (10) it follows that

$$
\begin{array}{ll}
f(n) \geqq f\left(R_{r-1}\right) \geqq(f(a))^{r-1} \geqq(f(a))^{\log _{a} n-2} & (a>1), \\
f(n) \leqq f\left(S_{r+2}\right) \leqq(f(a))^{r+2} \leqq(f(a))^{\log _{a} n+2} & (a>2) .
\end{array}
$$

Since (11) and (12) hold for all $a>2$, we have, for $a, b>2$,

$$
f(a)^{1 / \log a+2 / \log n} \geqq f(n)^{1 / \log n} \geqq f(b)^{1 / \log b-2 / \log n} .
$$

Since $n$ is arbitrary, this implies

$$
f(a)^{1 / \log a} \geqq f(b)^{1 / \log b} \quad(a, b>2) .
$$

But $a$ and $b$ are interchangeable in the above argument, so that

$$
f(a)^{1 / \log a}=f(b)^{1 / \log b} \quad(a, b>2) .
$$

Hence for $n>2, f(n)^{1 / \log n}$ is a constant. If this constant is 0 then $f(n) \equiv 0$, otherwise the constant can be written as $e^{k}$, and

$$
f(n)=n^{k} \quad(n>2) .
$$

From (1) it is clear that $f(1)=1$. Using $f(6)=f(2) f(3)$, we see from (16) that the result also holds for $n=2$; thus the proof is complete.

Research Institute of Canadian Mathematical Congress 